\title{
Electronic Cigarettes Efficacy and Safety at 12 Months: Cohort Study
}

\author{
Lamberto Manzoli ${ }^{1,2,3,40} *$, Maria Elena Flacco ${ }^{1,20}$, Maria Fiore ${ }^{5}$, Carlo La Vecchia ${ }^{6}$, \\ Carolina Marzuillo ${ }^{7}$, Maria Rosaria Gualano ${ }^{8}$, Giorgio Liguori ${ }^{9}$, Giancarlo Cicolini ${ }^{10}$, \\ Lorenzo Capasso ${ }^{1,3}$, Claudio D'Amario ${ }^{2}$, Stefania Boccia ${ }^{11}$, Roberta Siliquini ${ }^{8}$, \\ Walter Ricciardi ${ }^{11,12}$, Paolo Villari ${ }^{7}$
}

1 Department of Medicine and Aging Sciences, University of Chieti, via dei Vestini 5, 66013, Chieti, Italy, 2 Local Health Unit of Pescara, Via Renato Paolini 47, 65100, Pescara, Italy, 3 "University G. d'Annunzio" Foundation, Via Colle dell'Ara, 66013, Chieti, Italy, 4 Regional Healthcare Agency of Abruzzo, Via Attilio Monti 9, 65127, Pescara, Italy, 5 Department "G. F. Ingrassia"- Hygiene and Public Health, University of Catania, Piazza Università 2, 95131, Catania, Italy, 6 Department of Clinical Sciences and Community Health, University of Milan, Via Vanzetti 5, 20133, Milan, Italy, 7 Department of Public Health and Infectious Diseases, Sapienza University of Rome, Viale Regina Elena 324, 00161, Roma, Italy, 8 Department of Public Health Sciences, University of Turin, Via Santena 5/bis, Turin, 10124, Italy, 9 Department of Movement Sciences and Wellbeing, University Parthenope of Neaples, Via Medina 40, 80133, Napoli, Italy, 10 Local Health Authority of Lanciano-Vasto-Chieti, Via Martiri Lancianesi, 66100, Chieti, Italy, 11 Institute of Public Health, Università Cattolica del Sacro Cuore, Rome, Largo Francesco Vito, 1, 00168, Roma, Italy, 12 Italian Institute of Health, Viale Regina Elena 299, 00161, Rome, Italy

ه These authors contributed equally to this work.

* Imanzoli@post.harvard.edu

\section{G open ACCEss}

Citation: Manzoli L, Flacco ME, Fiore M, La Vecchia C, Marzuillo C, Gualano MR, et al. (2015) Electronic Cigarettes Efficacy and Safety at 12 Months: Cohort Study. PLoS ONE 10(6): e0129443. doi:10.1371/ journal.pone. 0129443

Academic Editor: Raymond Niaura, Legacy, Schroeder Institute for Tobacco Research and Policy Studies, UNITED STATES

Received: February 20, 2015

Accepted: May 9, 2015

Published: June 10, 2015

Copyright: @ 2015 Manzoli et al. This is an open access article distributed under the terms of the Creative Commons Attribution License, which permits unrestricted use, distribution, and reproduction in any medium, provided the original author and source are credited.

Data Availability Statement: Due to imposed ethical restrictions related to Italian privacy legislation, all relevant data are available upon request to Lambreto Manzoli ( Imanzoli@post.harvard.edu).

Funding: The authors have no support or funding to report.

Competing Interests: The authors have declared that no competing interests exist.

\section{Abstract}

\section{Objective}

To evaluate the safety and efficacy as a tool of smoking cessation of electronic cigarettes (e-cigarettes), directly comparing users of e-cigarettes only, smokers of tobacco cigarettes only, and smokers of both.

\section{Design}

Prospective cohort study. Final results are expected in 2019, but given the urgency of data to support policies on electronic smoking, we report the results of the 12-month follow-up.

\section{Data Sources}

Direct contact and structured questionnaires by phone or via internet.

\section{Methods}

Adults (30-75 years) were included if they were smokers of $\geq 1$ tobacco cigarette/day (tobacco smokers), users of any type of e-cigarettes, inhaling $\geq 50$ puffs weekly (e-smokers), or smokers of both tobacco and e-cigarettes (dual smokers). Carbon monoxide levels were tested in a sample of those declaring tobacco smoking abstinence. 


\section{Main Outcome Measures}

Sustained smoking abstinence from tobacco smoking at 12 months, reduction in the number of tobacco cigarettes smoked daily.

\section{Data Synthesis}

We used linear and logistic regression, with region as cluster unit.

\section{Results}

Follow-up data were available for 236 e-smokers, 491 tobacco smokers, and 232 dual smokers (overall response rate 70.8\%). All e-smokers were tobacco ex-smokers. At 12 months, $61.9 \%$ of the e-smokers were still abstinent from tobacco smoking; $20.6 \%$ of the tobacco smokers and $22.0 \%$ of the dual smokers achieved tobacco abstinence. Adjusting for potential confounders, tobacco smoking abstinence or cessation remained significantly more likely among e-smokers (adjusted OR 5.19; 95\% Cl: 3.35-8.02), whereas adding ecigarettes to tobacco smoking did not enhance the likelihood of quitting tobacco and did not reduce tobacco cigarette consumption. E-smokers showed a minimal but significantly higher increase in self-rated health than other smokers. Non significant differences were found in self-reported serious adverse events (eleven overall).

\section{Conclusions}

Adding e-cigarettes to tobacco smoking did not facilitate smoking cessation or reduction. If e-cigarette safety will be confirmed, however, the use of e-cigarettes alone may facilitate quitters remaining so.

\section{Registration Number}

NCT01785537.

\section{Introduction}

The electronic cigarette (e-cigarette) market has been growing sharply, reaching approximately $\$ 3$ billion in 2013 worldwide [1]. Despite the broad public health relevance, the published evidence on e-cigarettes safety and efficacy in reducing traditional tobacco cigarette smoking is limited to two randomized trials [2,3], two single-arm small trials [4-6], and six observational studies [7-12]. These studies mostly included smokers of both tobacco and e-cigarettes followed for 12 months or less, used various assessment methods, and reported controversial findings. Moreover, no direct comparisons between users of e-cigarettes only and smokers of tobacco cigarettes only are yet available [13].

In 2013 we started a 5-year study aimed at evaluating the long-term effects of e-cigarettes and to directly compare tobacco and e-cigarette exclusive smokers [14]. The final results are expected in 2019, but given the urgency of data to support policies on electronic smoking [1, 13, 15-17], we report here the main results of the 12-month follow-up. 


\section{Methods}

The protocol of this prospective cohort study has been reported elsewhere ([14], S1 File) and registered in Clinicaltrials.gov (NCT01785537). From June to November 2013 we recruited subjects through direct contact with general practitioners and e-cigarette shops, via internet advertisement and social networks. All participants were categorized to one of three natural and self-selected groups: tobacco smokers (if subjects had smoked $\geq 1$ tobacco cigarette per day for the past 6 months); e-smokers (if subjects had been smokers of any type of e-cigarettes, inhaling $\geq 50$ puffs weekly for the past 6 months); dual smokers (if subjects had smoked both tobacco and e-cigarettes within the same week for the past 6 months). Exclusion criteria were: age $<30 \mathrm{y}$ and $>75 \mathrm{y}$; pregnancy or breastfeeding; illicit drug use, major depression, severe allergies, angina, and past episodes of smoking-related major diseases [14]. We originally recruited all volunteers and planned to check after two months from the start whether the distribution of smokers was largely unbalanced (given the much higher proportion of tobacco smokers only in the Italian population). At the 2-month check, we realized that the number of tobacco smokers only already exceeded the requested sample, and stopped their recruitment.

Data were collected through a structured questionnaire on smoking habits, previous and current diseases, lifestyle behaviour, and quality of life. The same questionnaire was administered through phone interview and/or by internet (www.ipazienti.it/fumo) after 12 months, and follow-up is scheduled to continue up to 60 months. Two investigators (MEF and LM) tested carbon monoxide levels in expired after breath (Smokerlyzer piCO+, Bedfont Scientific Ltd.) in a $25 \%$ random sample of those declaring tobacco smoking abstinence at the end of follow-up.

The primary outcome was the percentage of subjects reporting sustained (30 days) smoking abstinence from tobacco smoking at 12 months. Other outcomes were the proportion of quitters from all types of smoking (tobacco and e-cigarettes), the number of tobacco cigarettes smoked, self-reported health, and serious adverse events. Linear and logistic regressions, with region as cluster unit, were used to investigate potential predictors of continuous and categorical outcomes, respectively. Details on data analysis are reported in S2 File (web appendix).

The work was approved by the Ethics Committee of the University of Chieti (Record n. 6; 25-03-2013). All participants provided their written informed consent to participate in the study.

\section{Protocol violations}

The study was originally planned to be funded, but the sponsor withdrew when the protocol was already approved and the study started. Therefore, the following deviations from the original protocol occurred due to funding limitations: (1) the sample was smaller than originally planned (500 subjects per group); (2) the 6-month follow-up interview was not made; (3) carbon monoxide levels were not tested in all quitters and non-relapsing e-smokers but only in a $25 \%$ random sample of them. Adverse events data will be checked for the residents in Abruzzo ( $46.1 \%$ of the sample) through data linkage with hospital and pharmaceutical administrative databases, but such datasets are typically provided with delay and could not be available at the first follow-up.

\section{Results}

The flow of the participants is shown in Fig 1. Data at twelve months were available for $236 \mathrm{e}-$ smokers, 491 tobacco smokers, and 232 dual smokers. Overall, the mean age was $44.5 \pm 11.6$ years, and $55.9 \%$ were males. Few differences in baseline characteristics were found between participants completing the 12-month phase and withdrawals or lost to follow-up 


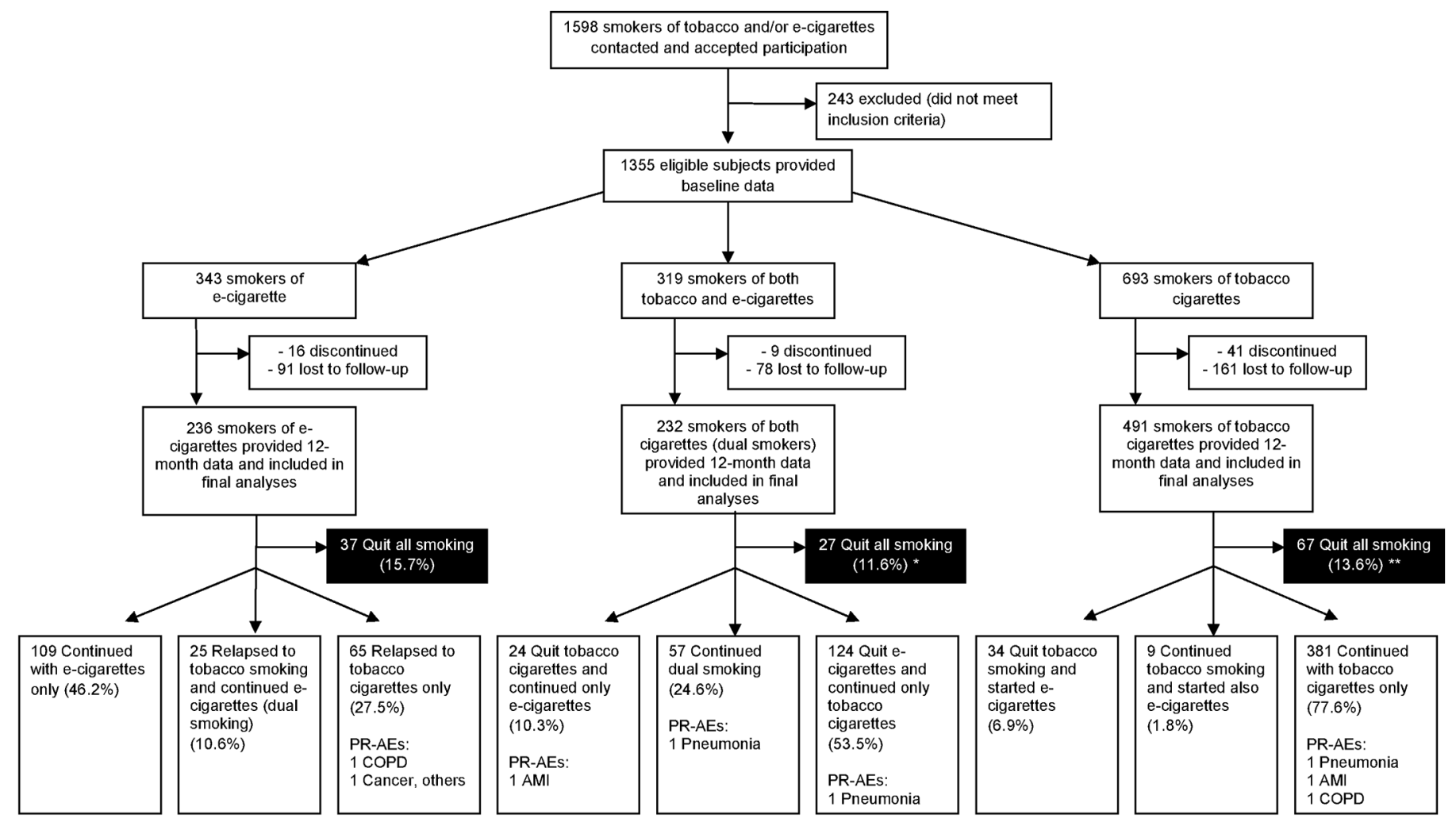

PR-AEs: Possibly related adverse events. AMI = Acute Myocardial infarction. COPD = Chronic obstructive pulmonary diseases. * 1 COPD episode. ${ }^{*} 1 \mathrm{Pneumonia}, 1 \mathrm{AMI}$, and 1 angina.

Fig 1. Flow of the participants and numbers of quitters and serious adverse events.

doi:10.1371/journal.pone.0129443.g001

(29.2\%; S1 Table), as well as among the three types of smokers (Table 1). All e-cigarette users were former tobacco smokers, since more than 20 years on average.

After twelve months, $61.9 \%$ of the e-smokers were still abstinent from tobacco smoking; $20.6 \%$ of the tobacco smokers and $22.0 \%$ of the dual smokers achieved tobacco abstinence (Table 2). More than half (53.5\%) of dual smokers abandoned e-cigarettes and continued to smoke only tobacco cigarettes (Fig 2). The proportion of subjects who quit all types of smoking (tobacco and e-cigarettes) did not significantly differ by baseline smoking status: 15.7\%, 13.7\% and $11.6 \%$ among electronic, tobacco and dual smokers, respectively $(\mathrm{p}>0.05)$. If analyzed with an Intention-To-Treat approach, which is however problematic given the large amount of switchers in the study, the above proportions of all-smoking quitters were $10.8 \%, 8.7 \%$ and $9.5 \%$, respectively (all $\mathrm{p}>0.05)$. Only $41(8.8 \%)$ e-smokers used nicotine-free e-cigarettes, and they were similar to nicotine e-smokers on both the rates of tobacco smoking relapse $(43.9 \%$ vs $40.3 \%$, respectively) and the rates of all-smoking cessation ( $14.6 \%$ vs $13.8 \%$, respectively; all $\mathrm{p}>0.05)$.

Of the 154 subjects initially declaring tobacco smoking cessation during follow-up, and of the 147 e-smokers declaring prolonged tobacco abstinence, 38 and 36 underwent a test to detect exhaled CO levels, respectively. CO levels suggestive of tobacco smoking ( $>7 \mathrm{ppm}$ ) [18] were found in only three subjects (two tobacco smokers and one e-smoker), who admitted the error and were accordingly re-classified.

The results on the change in tobacco cigarette consumption from baseline were controversial. On one side, the percentage of subjects who reduced the number of tobacco cigarettes 
smoked per day by $50 \%$ or more from baseline was similar among dual and tobacco only smokers (29.2\% and $29.4 \%$, respectively). On the other side, the mean daily consumption of tobacco cigarette varied widely according to 12-month smoking status (Table 2): among dual smokers,

Table 1. Baseline characteristics of the subjects completing the 12-month follow-up.

\begin{tabular}{|c|c|c|c|c|c|}
\hline \multirow{3}{*}{ Variables } & \multicolumn{3}{|c|}{ Baseline smoking status } & \multirow{3}{*}{$\frac{\text { Overall }}{(N=959)}$} & \multirow[b]{3}{*}{$p^{*}$} \\
\hline & E-cigarettes only & Tobacco cigarettes only & Dual smoking & & \\
\hline & $(n=236)$ & $(n=491)$ & $(n=232)$ & & \\
\hline Mean age in years (SD) & $45.2(10.7)$ & $44.2(11.9)$ & $44.3(12.0)$ & $44.5(11.6)$ & \\
\hline Male gender, \% & 62.7 & 48.7 & 64.2 & 55.9 & $*, * *$ \\
\hline Mean BMI (SD) & 24.7 (3.9) & $24.4(4.0)$ & $24.8(4.0)$ & $24.6(4.0)$ & \\
\hline Married, \% & 60.5 & 54.8 & 55.7 & 56.4 & \\
\hline Employed, \% & 78.9 & 79.5 & 74.4 & 78.2 & \\
\hline \multicolumn{6}{|l|}{ Educational level, \% } \\
\hline - Elementary / Middle & 21.9 & 21.9 & 22.1 & 22.4 & \\
\hline - High school & 54.5 & 42.5 & 46.7 & 46.3 & * \\
\hline - Bachelor or higher & 23.6 & 35.6 & 31.2 & 31.3 & $*$ \\
\hline \multicolumn{6}{|l|}{ Physical activity (69 missing) } \\
\hline - At work, \% & 18.9 & 20.3 & 15.7 & 18.9 & \\
\hline - Weekly hours at work, mean (SD) & $23.8(18.4)$ & $26.7(16.6)$ & $22.9(19.0)$ & $25.3(17.5)$ & \\
\hline - At home, \% & 48.0 & 48.0 & 51.3 & 48.8 & \\
\hline - Weekly hours at home, mean (SD) & $5.3(4.7)$ & $5.2(5.5)$ & $5.3(4.5)$ & $5.3(5.1)$ & \\
\hline \multicolumn{6}{|l|}{ Alcohol use } \\
\hline Regular alcohol intake, \% & 20.0 & 29.4 & 27.4 & 26.6 & * \\
\hline Mean alcohol units daily (SD) & $2.1(1.2)$ & $2.1(1.6)$ & $2.1(1.0)$ & $2.1(1.4)$ & \\
\hline \multicolumn{6}{|l|}{ Cardiovascular risk and health } \\
\hline - Hypertension, \% & 13.6 & 11.6 & 9.9 & 11.7 & \\
\hline - Diabetes, \% & 4.2 & 3.3 & 4.3 & 3.8 & \\
\hline - Hypercholesterolemia, \% & 8.1 & 8.8 & 10.3 & 9.0 & \\
\hline - Self-reported health, mean (SD) ${ }^{€}$ & $8.0(1.3)$ & $7.8(1.3)$ & $7.7(1.2)$ & $7.8(1.3)$ & \\
\hline - Low $(<6)$ self-reported health $€$, \% & 5.0 & 5.5 & 3.3 & 4.9 & \\
\hline \multicolumn{6}{|l|}{ Smoking pattern, mean (SD) } \\
\hline - Years of tobacco smoking & $21.4(10.7)^{¥}$ & $22.3(12.6)$ & $25.2(12.5)$ & $22.9(12.1)$ & $* * * * *$ \\
\hline - N. tobacco cigarettes daily & - & $14.1(8.1)$ & $14.9(9.8)$ & $14.4(8.7)$ & \\
\hline - Months of electronic smoking & $8.8(5.1)$ & - & $8.4(4.5)$ & $8.6(4.8)$ & \\
\hline - N. e-cigarette daily puffs & $162(276)$ & - & $96(146)$ & $130(224)$ & $* * *$ \\
\hline - EC nicotine dose in $\mathrm{mg}$ & $8.7(5.2)$ & - & $10.9(5.6)$ & $9.8(5.5)$ & $* * *$ \\
\hline \multicolumn{6}{|l|}{ E-cigarettes by nicotine dose, $\%$} \\
\hline - No nicotine & 12.8 & - & 5.6 & 9.3 & $* * *$ \\
\hline - 3 to $8 \mathrm{mg}$ & 23.5 & - & 19.1 & 21.3 & \\
\hline$-9 \mathrm{mg}$ & 40.7 & - & 34.0 & 37.4 & \\
\hline-10 to $24 \mathrm{mg}$ & 23.0 & & 41.4 & 32.0 & $* * *$ \\
\hline - Former tobacco smoking, \% & 100.0 & 100.0 & 100.0 & 100.0 & \\
\hline - Use of other tobacco products ${ }^{\psi}, \%$ & 0.8 & 0.4 & 0.9 & 0.6 & \\
\hline - Use of other nicotine products ${ }^{\Omega}, \%$ & 0.4 & 0.0 & 0.0 & 0.1 & \\
\hline \multicolumn{6}{|l|}{ Reasons of e-cigarette smoking ${ }^{\varphi}$} \\
\hline - Stop tobacco smoking, \% & 74.1 & - & 45.7 & 60.0 & $* * *$ \\
\hline
\end{tabular}

(Continued) 
Table 1. (Continued)

\begin{tabular}{|c|c|c|c|c|c|}
\hline \multirow{3}{*}{ Variables } & \multicolumn{3}{|c|}{ Baseline smoking status } & \multirow{3}{*}{$\begin{array}{l}\text { Overall } \\
(N=959)\end{array}$} & \multirow[b]{3}{*}{$p^{*}$} \\
\hline & E-cigarettes only & Tobacco cigarettes only & Dual smoking & & \\
\hline & $(n=236)$ & $(n=491)$ & $(n=232)$ & & \\
\hline - Reduce tobacco smoking, \% & 16.9 & - & 56.5 & 36.5 & $* * *$ \\
\hline - Indoor smoking, \% & 16.1 & - & 12.1 & 14.1 & \\
\hline \multicolumn{6}{|c|}{$€$ EuroQol final question, ranging from 1 (feel very bad) to 10 (perfectly healthy). This item had 56 missing values. } \\
\hline \multicolumn{6}{|c|}{$\Psi$ Cigars or tobacco chewing. } \\
\hline \multicolumn{6}{|l|}{$\Omega$ Nicotine patch or gums. } \\
\hline \multicolumn{6}{|l|}{${ }^{\varphi}$ More than one answer allowed. } \\
\hline \multicolumn{6}{|c|}{$¥$ Years of former tobacco smoking for e-cigarette only smokers. } \\
\hline \multicolumn{6}{|l|}{$\mathrm{P}<0.01$ for the comparison } \\
\hline \multicolumn{6}{|c|}{ * Tobacco only vs electronic cigarettes only } \\
\hline \multicolumn{6}{|c|}{ ** Tobacco only vs dual smoking } \\
\hline *** E-cigarettes only vs dual si & & & & & \\
\hline
\end{tabular}

doi:10.1371/journal.pone.0129443.t001 
Table 2. Main outcomes at twelve months.

\begin{tabular}{|c|c|c|c|c|}
\hline & \multicolumn{3}{|c|}{ Baseline smoking status } & \multirow[b]{3}{*}{$p^{*}$} \\
\hline & $\begin{array}{l}\text { E-cigarettes } \\
\text { only }\end{array}$ & $\begin{array}{l}\text { Tobacco } \\
\text { cigarettes only }\end{array}$ & $\begin{array}{l}\text { Dual } \\
\text { smoking }\end{array}$ & \\
\hline & $(n=236)$ & $(n=491)$ & $(n=232)$ & \\
\hline \multicolumn{5}{|l|}{ 1. Smoking status } \\
\hline \multicolumn{5}{|l|}{ Tobacco smoking, \% (n) } \\
\hline - Tobacco smoking continuous abstinence or cessation, \% (n) & $61.9(146)$ & $20.6(101)$ & $22.0(51)$ & $*$, *** \\
\hline - Tobacco smoking (continued or relapsed) & $38.1(90)$ & $79.4(390)$ & $78.0(181)$ & $*$, *** \\
\hline \multicolumn{5}{|l|}{ All types of smoking, \% (n) } \\
\hline - Quit all smoking (tobacco and e-cigarettes) & $15.7(37)$ & $13.7(67)$ & $11.6(27)$ & \\
\hline - E-cigarettes & $46.2(109)$ & $6.9(34)$ & $10.3(24)$ & $*$, *** \\
\hline - Both (mixed) & $10.6(25)$ & $1.8(9)$ & $24.6(57)$ & $\begin{array}{l}*, * * \\
* * *\end{array}$ \\
\hline - Tobacco cigarettes & $27.5(65)$ & $77.6(381)$ & $53.5(124)$ & $\stackrel{* * *}{* *}$, \\
\hline \multicolumn{5}{|l|}{$\begin{array}{l}\text { 2. Number of tobacco cigarettes-mean difference in the daily } n \text {. between } 12 \\
\text { months and baseline (SD) }\end{array}$} \\
\hline Stratified by smoking status at baseline & - & $-3.1(7.5)$ & $-2.9(11.6)$ & 0.7 \\
\hline \multicolumn{5}{|l|}{ Stratified by smoking status at 12 months } \\
\hline - Those who continued with the same type of smoking (no switch) & - & $-1.5(6.1)$ & $-4.9(10.8)$ & 0.001 \\
\hline $\begin{array}{l}\text { - Switched to tobacco cigarettes only (for those initially smoking both) or to both (for } \\
\text { those initially smoking tobacco cigarettes only) }\end{array}$ & - & $-6.2(9.0)$ & $+0.7(9.9)$ & 0.045 \\
\hline \multicolumn{5}{|l|}{ 3. Self-rated health ${ }^{€}$ - mean difference between 12 months and baseline (SD) } \\
\hline Stratified by smoking status at baseline & $+0.3(1.5)$ & $0.0(1.5)$ & $+0.1(1.7)$ & $*{ }^{* * *}$ \\
\hline \multicolumn{5}{|l|}{ Stratified by smoking status at 12 months } \\
\hline - Quit all smoking & $+0.3(1.4)$ & $+0.2(2.0)$ & $+0.7(1.7)$ & \\
\hline - Smoking e-cigarettes only & $+0.5(1.3)$ & $+0.9(2.1)$ & $+1.0(1.4)$ & \\
\hline - Smoking both tobacco and e-cigarettes & $+0.6(1.3)$ & $-0.1(1.7)$ & $+0.1(1.5)$ & \\
\hline - Smoking tobacco cigarettes only & $-0.4(1.6)$ & $-0.1(1.4)$ & $-0.1(1.8)$ & \\
\hline \multicolumn{5}{|l|}{ 4. Safety-possibly-related serious adverse events \% (n) ${ }^{\psi}$} \\
\hline Stratified by smoking status at baseline & $0.9(2)$ & $1.2(6)$ & $1.7(4)$ & \\
\hline \multicolumn{5}{|l|}{ Stratified by smoking status at 12 months } \\
\hline - Quit all smoking & 0.0 & $4.5(3)$ & $3.7(1)$ & \\
\hline - Smoking e-cigarettes only & 0.0 & 0.0 & $4.2(1)$ & \\
\hline - Smoking both tobacco and e-cigarettes & 0.0 & 0.0 & $1.8(1)$ & \\
\hline - Smoking tobacco cigarettes only & $3.1(2)$ & $0.8(3)$ & $0.8(1)$ & \\
\hline
\end{tabular}

SD $=$ Standard deviation .

$\mathrm{P}<0.01$ for the comparison

* Tobacco only vs electronic cigarettes only

** Tobacco only vs both tobacco and electronic cigarettes (dual smoking)

*** E-cigarettes only vs both tobacco and electronic cigarettes (dual smoking).

$€$ EuroQol final item, ranging from 1 (feel very bad) to 10 (perfectly healthy).

${ }^{\Psi}$ Chronic obstructive pulmonary diseases, stroke, heart failure, myocardial infarction, angina, pneumonia, cancer of: larynx or oral cavity, lung, stomach, pancreas, cervix, kidney, bladder, myeloid leukaemia.

doi:10.1371/journal.pone.0129443.t002

increase; $\mathrm{p}=0.027$ ), as it was diabetes mellitus (coeff. $-0.82 ; \mathrm{p}=0.010$ ). Only the daily number of tobacco cigarettes at baseline was significantly, inversely associated with their reduction during follow-up (coeff. 0.56 for each cigarette increase; $\mathrm{p}<0.001$ ). 
Smokers of e-cigarettes (e-cig) only after 12 months $(n=236)$

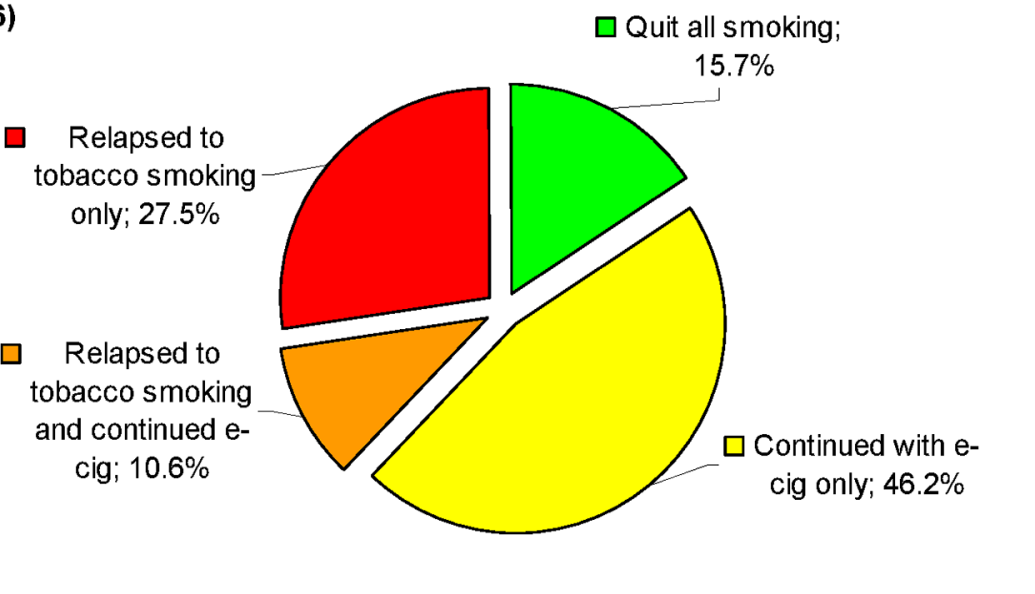

\section{Smokers of tobacco cigarettes only} after 12 months $(n=491)$

$\square$ Quit all smoking; $13.7 \%$

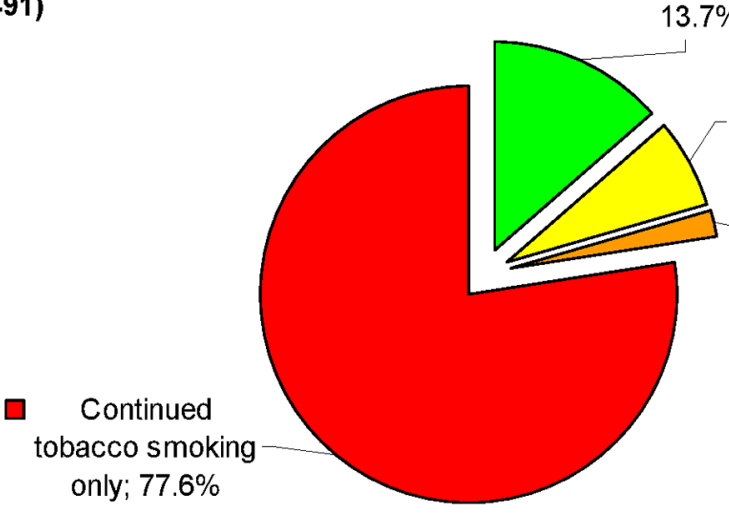

$\square$ Quit tobacco and switched to e-cig only; $6.9 \%$

$\square$ Started also ecig; $1.8 \%$
Smokers of both tobacco and e-cigarettes after 12 months ( $n=232)$ $\square$ Quit all smoking; $11.6 \%$

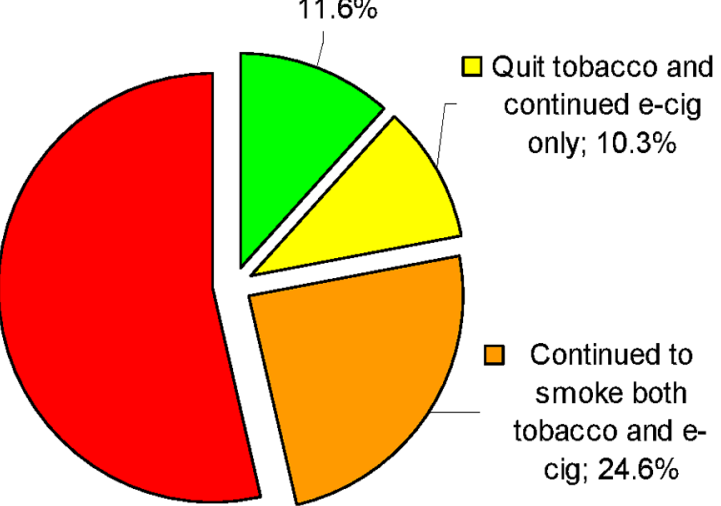

Fig 2. Smoking status after twelve months of follow-up, by smoking status at baseline.

doi:10.1371/journal.pone.0129443.g002 
Table 3. Smoking abstinence and cessation, difference in the number of daily cigarette smoked and self-reported health: results of the multivariate analyses.

\begin{tabular}{|c|c|c|c|c|}
\hline & Adjusted OR & $\mathbf{p}$ & Crude OR & $\mathbf{p}$ \\
\hline Outcomes at 12 month & $(95 \% \mathrm{Cl})$ & & $(95 \% \mathrm{Cl})$ & \\
\hline \multicolumn{5}{|c|}{ Tobacco smoking continuous abstinence or cessation ${ }^{A}$} \\
\hline Tobacco cigarettes only (ref. cat.) & 1 & - & 1 & - \\
\hline E-cigarettes only & $5.19(3.35 ; 8.02)$ & $<0.001$ & $5.03(3.41 ; 7.40)$ & $<0.001$ \\
\hline Both tobacco and e-cigarettes & $0.83(0.53 ; 1.29)$ & 0.4 & $0.93(0.62 ; 1.40)$ & 0.7 \\
\hline \multicolumn{5}{|l|}{ All smoking cessation ${ }^{A}$} \\
\hline Tobacco cigarettes only (ref. cat.) & 1 & - & 1 & - \\
\hline E-cigarettes only & $1.25(0.77 ; 2.04)$ & 0.4 & $1.18(0.76 ; 1.82)$ & 0.5 \\
\hline \multirow[t]{3}{*}{ Both tobacco and e-cigarettes } & $0.70(0.41 ; 1.19)$ & 0.2 & $0.83(0.52 ; 1.34)$ & 0.5 \\
\hline & Adjusted coefficient & & Raw coefficient & \\
\hline & $(95 \% \mathrm{Cl})$ & & $(95 \% \mathrm{Cl})$ & \\
\hline \multicolumn{5}{|c|}{ Difference in the daily $n$. or tobacco cigarettes from 12 months to baseline ${ }^{B}$} \\
\hline Tobacco cigarettes only (ref. cat.) & 0 & - & 0 & - \\
\hline Both tobacco and e-cigarettes & $0.60(-0.70 ; 1.91)$ & 0.4 & $0.28(-1.23 ; 1.78)$ & 0.7 \\
\hline \multicolumn{5}{|c|}{ Difference in the self-reported health score from 12 months to baseline ${ }^{C}$} \\
\hline Tobacco cigarettes only (ref. cat.) & 0 & - & 0 & - \\
\hline E-cigarettes only & $0.31(0.04 ; 0.59)$ & 0.026 & $0.25(0.00 ; 0.50)$ & 0.047 \\
\hline Both tobacco and e-cigarettes & $0.14(-0.13 ; 0.40)$ & 0.3 & $0.13(-0.13 ; 0.39)$ & 0.3 \\
\hline
\end{tabular}

OR = Odds Ratio. $\mathrm{Cl}=$ Confidence Interval.

A Random-effect logistic regression with region as the cluster level, adjusting for the following baseline characteristics: age, gender, BMI, marital status, educational level, occupation, alcohol use, hypertension, hypercholesterolemia, diabetes, self-reported health, years of tobacco smoking (former smoking for e-cigarette users), $n$. of tobacco cigarettes smoked per day (or puffs per day for e-cigarette only smokers). 903 subjects were included in the final model due to 56 missing items in the self-reported health item at baseline.

${ }^{B}$ E-cigarette only smokers were not included. Random-effect linear regression with region as the cluster level, adjusting for the following baseline characteristics: age, gender, BMI, marital status, educational level, occupation, alcohol use, hypertension, hypercholesterolemia, diabetes, self-reported health, years of tobacco smoking, n. of tobacco cigarettes smoked per day. 685 subjects were included in the final model due to 38 missing items in the self-reported health item at baseline.

${ }^{\mathrm{C}}$ Random-effect linear regression with region as the cluster level, adjusting for the following baseline characteristics: age, gender, BMI, marital status, educational level, occupation, alcohol use, hypertension, hypercholesterolemia, diabetes, self-reported health, years of tobacco smoking (former smoking for e-cigarette users), n. of tobacco cigarettes smoked per day (or puffs per day for e-cigarette only smokers). 874 subjects were included in the final model due to 56 missing items in the self-reported health item at baseline and 29 missing items in the self-reported health at twelve months.

doi:10.1371/journal.pone.0129443.t003

\section{Discussion}

E-cigarettes are a controversial issue. Some experts suggested that e-cigarette use should be restricted or banned [19] because of their potential to increase tobacco cigarette consumption (reducing motivation to completely quit, acting as a gateway to smoking for non-smokers, or increasing smoking social acceptability) [19-25], and because concerns have been raised on the potential harms from propylene glycol [19,24], cartridge manufacturing and content quality (as refills may contain toxins and cause nicotine poisoning) [16, 19,24], and on the potential risks from second-hand electronic smoking in indoor environments [15, 19]. On the contrary, other experts claimed that e-cigarettes may be the centrepiece of a harm reduction strategy (being more attractive and cheap than other nicotine replacement therapies and facilitating smoking cessation, reduction or abstinence), that there is no evidence of undesirable uptake from non-smokers, that e-cigarettes pose only a small fraction of the risks of tobacco cigarettes (as tested liquids and aerosols contain negligible concentrations of toxicants and carcinogens, 
and use of nicotine without tobacco toxicants poses little risks for most of the population) [16, 25-28]. They therefore suggest that no regulations other than quality controls should be introduced, in order to encourage smokers to switch to a safer form of addiction [16, 27]. At least, there is universal consensus that current evidence is scarce and long-term data on e-cigarette safety and efficacy are urgently needed $[16,19-23,25,29,30]$.

This is the first study directly comparing smokers of tobacco cigarettes only with users of ecigarettes only, and aimed at providing safety data on the largest sample of e-cigarette users for the longest time. On one side, the use of e-cigarettes in addition to tobacco (dual smoking) did not seem to improve neither smoking cessation nor reduction: as compared with tobacco only smokers, dual smokers showed a similar quitting rate, no difference in self-rated health, and a non-significant reduction in the number of cigarettes smoked daily. On the other side, no safety concerns raised during the study, although the limitations in adverse events recording prevent us to draw any conclusions. The users of e-cigarettes only showed a minimal though significant increase in self-rated health and, most importantly, they showed a 1-year relapse rate which is relatively low (38.1\%) when compared with real-life rates (60\% to $90 \%)$ of tobacco-cigarette smokers [31]. Indeed, such a low relapse rate could be partially explained by the fact that, in this sample, the users of e-cigarettes only did quit tobacco smoking already when the study started (since 8 months on average). Thus, the high proportion of e-cigarette users who did not relapse (that is, remained quitters) cannot be interpreted tout-court as a smoking cessation rate. Given this, if e-cigarettes will be proved safer than tobacco cigarettes, this finding may still support the utility of e-cigarettes as a tool to help quitters remaining so. Clearly, its interpretation would be drastically different if e-cigarettes would prove to be equally or even less safe than tobacco cigarettes: although unlikely, in such a case the high adherence to e-cigarette use would be an extremely negative finding, eventually supporting more restrictive regulations and/or a complete ban.

Current data on serious adverse events in e-cigarette users are limited. Only three studies evaluated such an outcome on healthy subjects $[2,3,6]$. Moreover, in one study the intervention with e-cigarettes lasted 3 months [3], and in the other two studies those who continued ecigarette use for six months were, in total, 27 [5] and 95 (one third of the sample excluded, protocol violation $\mathrm{n}$. 1) [2]. Thus, although the methodology of the above studies was rigorous, so far the entire evidence on e-cigarette safety at 6 months is limited to 122 subjects, most of whom were also smoking tobacco for most of the follow-up. In the present study, data are provided on 134 baseline e-smokers only and 81 dual smokers who continued to use e-cigarettes for twelve months. We observed a low rate of serious adverse events, with no differences by smoking status. Safety data were self-reported and it was not possible to establish whether such events may be related to smoking. Also, considering that also our participants were past tobacco smokers, even among the smokers of e-cigarettes only some more reliable information will be available exclusively in the next years of follow-up, when also administrative data will be available to check self-reported data. Therefore, although the present results agree with previous studies [2, 3, 5] and FDA Center for Tobacco Products data [32] suggesting no increase in health risks with e-cigarette use, they do not allow to draw any firm conclusion and more research is strongly needed on electronic smoking safety, which remains the most important issue from a public health perspective.

Our findings on smoking cessation and reduction rates of tobacco smokers are comparable to those by Choi et al. [9] and Adkinson et al. [8], slightly higher than those reported by Grana et al. among smokers unwilling to quit [10], and lower than the highest $31.3 \%$ quit rate reported by Vickerman et al. among quit-line callers [11]. Smoking reduction outcomes in our dual smokers were also similar to most reported data $[5,6,8]$, and higher than those reported by Caponnetto et al. among smokers of $\geq 10$ cigarettes/day unwilling to quit [3]. Our smoking 
cessation rate in dual smokers was however comparable or lower than that from three studies only $[7,11,12]$ and higher than several other studies $[3,6,8-10]$. As a potential explanation, three of such studies may have had less motivated samples (smokers unwilling to quit) $[3,6$, 10], and another included countries with an e-cigarette ban and was made during previous years (data collected from 2008 to 2011), thus the motivation and/or the possibility to switch to e-cigarette only might have reduced [8]. Our relapse rate among the users of e-cigarettes only more than doubled the estimate by Etter et al. (whose sample, however, may have been highly motivated as recruitment started from smoking cessation websites [7]), but it was much lower than the $\cong 94 \%$ relapse rate documented in the RCT by Bullen et al. [2]. However, such data cannot be compared directly, as our observational design included e-cigarette users that were already spontaneous quitters since eight months on average, smoked any type of e-cigarette rather than those provided by investigators, and were not selected as smokers of $\geq 10$ cigarettes daily. In any case, such a discrepancy indicates that our low relapse rate among e-smokers should only be interpreted as the abstinence achieved by people who already quit, not as the smoking cessation rate that e-cigarette (alone) might have if used as an intervention among smokers who did not quit.

This study has some limitations. First, smoking cessation was mostly self-reported, as we were able to test carbon monoxide levels only in $25 \%$ of the sample in abstinence from tobacco smoking. In such a sample, however, only $4 \%$ of the quitters $(n=3)$ had to be reclassified. Second, we had no follow-up data for almost $30 \%$ of baseline participants, a loss rate lower than most previous prospective studies $[3,6,7,10-12]$ but still prone to bias. However, non-responders were similar to responders for most variables. In any case, those accepting to participate to the study and providing follow-up data are likely to be more motivated, which may partially explain the high smoking cessation rate even when e-cigarette switchers are excluded (13.7\%). As in previous Italian studies [3, 6], even if cessation rates are likely to be overestimated with respect to the Italian average population (www.istat.it), there are no reasons to believe that this should bias the differences by smoking status. Third, like all previous observational studies we included all types of e-cigarettes to approach real-life conditions, but different e-cigarette models with various nicotine doses might lead to diverse results. However, in this study the rates of tobacco abstinence and all-smoking cessation of the users of nicotine-free ecigarettes were similar to those of nicotine e-cigarette users. In agreement with previous data $[2,3,5]$, such finding deserves further investigation in order to better understand the role of rituals such as handling and manipulation in determining smoking dependence [3].

\section{Conclusions}

In conclusion, during the 12-month follow-up no serious safety concerns emerged among the largest sample of e-cigarette users examined so far. Such data, however, must be considered preliminary. Adding e-cigarettes to tobacco smoking did not facilitate smoking cessation or reduction, as dual smokers and tobacco only smokers showed similar outcomes. In contrast, most exclusive users of e-cigarettes were able to maintain smoking abstinence at twelve months. If e-cigarette safety will be confirmed in long-term evaluations including the present, they may be a promising tool to help quitters remain so.

\section{Supporting Information}

S1 File. Study protocol.

(PDF) 
S2 File. Outcome variables and data analysis.

(DOC)

S1 Table. Characteristics of the subjects completing the 12-month follow-up vs withdrawals or lost-to-follow-up subjects.

(DOC)

S2 Table. Secondary outcomes.

(DOC)

\section{Acknowledgments}

The investigators are grateful to Dr. Serena Di Sante, Giorgia Di Sante, Prof. Riccardo Polosa, Prof. Pasquale Caponnetto, students of the Faculty of Medicine of Catania and general physicians from Abruzzo (Francesco Di Silvestro, Luciano Giacci, Marino Mincone, Gabriella Salladini, Giuliano Salvio, Renato Seller, Lucio Zinni) for their help during recruitment.

\section{Author Contributions}

Conceived and designed the experiments: LM MEF CLV RS SB WR PV. Analyzed the data: LM MEF. Contributed reagents/materials/analysis tools: LM MEF. Wrote the paper: LM MEF. Participated in the design, analysis and interpretation of the study: LM MEF MF CLV CM MRG GL GC LC CD SB RS WR PV. Collected baseline and follow-up data, and assisted LM and MEF in data analysis: MF CM MRG GC GL CD LC. Tested CO levels: LM MEF. Guarantor for all data: LM.

\section{References}

1. WHO FCoTC. Electronic nicotine delivery systems. [August 29, 2014]. Available from: http://apps.who. int/gb/fctc/PDF/cop6/FCTC_COP6_10-en.pdf?ua=1.

2. Bullen $\mathrm{C}$, Howe $\mathrm{C}$, Laugesen $\mathrm{M}, \mathrm{McRobbie} \mathrm{H}$, Parag V, Williman J, et al. Electronic cigarettes for smoking cessation: a randomised controlled trial. Lancet. 2013; 382(9905):1629-37. Epub 2013/09/14. doi: 10.1016/S0140-6736(13)61842-5S0140-6736(13)61842-5 [pii]. PMID: 24029165.

3. Caponnetto P, Campagna D, Cibella F, Morjaria JB, Caruso M, Russo C, et al. EffiCiency and Safety of an eLectronic cigAreTte (ECLAT) as tobacco cigarettes substitute: a prospective 12-month randomized control design study. PLoS One. 2013; 8(6):e66317. Epub 2013/07/05. doi: 10.1371/journal.pone. 0066317PONE-D-13-05991 [pii]. PMID: 23826093; PubMed Central PMCID: PMC3691171.

4. Caponnetto P, Auditore R, Russo C, Cappello GC, Polosa R. Impact of an electronic cigarette on smoking reduction and cessation in schizophrenic smokers: a prospective 12-month pilot study. Int J Environ Res Public Health. 2013; 10(2):446-61. Epub 2013/01/30. doi: 10.3390/ ijerph10020446ijerph10020446 [pii]. PMID: 23358230; PubMed Central PMCID: PMC3635154.

5. Polosa R, Caponnetto P, Morjaria JB, Papale G, Campagna D, Russo C. Effect of an electronic nicotine delivery device (e-Cigarette) on smoking reduction and cessation: a prospective 6-month pilot study. BMC Public Health. 2011; 11:786. Epub 2011/10/13. doi: 10.1186/1471-2458-11-7861471-2458-11786 [pii]. PMID: 21989407; PubMed Central PMCID: PMC3203079.

6. Polosa R, Morjaria JB, Caponnetto P, Campagna D, Russo C, Alamo A, et al. Effectiveness and tolerability of electronic cigarette in real-life: a 24-month prospective observational study. Intern Emerg Med. 2014; 9(5):537-46. Epub 2013/07/23. doi: 10.1007/s11739-013-0977-z PMID: 23873169.

7. Etter JF, Bullen C. A longitudinal study of electronic cigarette users. Addict Behav. 2014; 39(2):491-4. Epub 2013/11/16. doi: 10.1016/j.addbeh.2013.10.028S0306-4603(13)00330-4 [pii]. PMID: 24229843.

8. Adkison SE, O'Connor RJ, Bansal-Travers M, Hyland A, Borland R, Yong HH, et al. Electronic nicotine delivery systems: international tobacco control four-country survey. Am J Prev Med. 2013; 44(3):20715. Epub 2013/02/19. doi: 10.1016/j.amepre.2012.10.018S0749-3797(12)00822-7 [pii]. PMID: 23415116 ; PubMed Central PMCID: PMC3627474.

9. Choi K, Forster JL. Authors' response. Am J Prev Med. 2014; 46(6):e58-9. Epub 2014/05/21. doi: 10. 1016/j.amepre.2014.02.013S0749-3797(14)00116-0 [pii]. PMID: 24842747. 
10. Grana RA, Popova L, Ling PM. A longitudinal analysis of electronic cigarette use and smoking cessation. JAMA Intern Med. 2014; 174(5):812-3. Epub 2014/03/26. doi: 10.1001/jamainternmed.2014. 1871846627 [pii]. PMID: 24664434; PubMed Central PMCID: PMC4122246.

11. Vickerman KA, Carpenter KM, Altman T, Nash CM, Zbikowski SM. Use of electronic cigarettes among state tobacco cessation quitline callers. Nicotine Tob Res. 2013; 15(10):1787-91. Epub 2013/05/10. doi: 10.1093/ntr/ntt061ntt061 [pii]. PMID: 23658395.

12. Biener L, Hargraves JL. A Longitudinal Study of Electronic Cigarette Use in a Population-Based Sample of Adult Smokers: Association With Smoking Cessation and Motivation to Quit. Nicotine Tob Res. 2014. Epub 2014/10/11. doi: ntu200 [pii]doi: 10.1093/ntr/ntu200 PMID: 25301815.

13. McRobbie H, Bullen $\mathrm{C}$, Hartmann-Boyce J, Hajek $\mathrm{P}$. Electronic cigarettes for smoking cessation and reduction. Cochrane Database Syst Rev. 2014; 12:CD010216. Epub 2014/12/18. doi: 10.1002/ 14651858.CD010216.pub2 PMID: 25515689.

14. Manzoli L, La Vecchia C, Flacco ME, Capasso L, Simonetti V, Boccia S, et al. Multicentric cohort study on the long-term efficacy and safety of electronic cigarettes: study design and methodology. BMC Public Health. 2013; 13:883. Epub 2013/09/26. doi: 10.1186/1471-2458-13-8831471-2458-13-883 [pii]. PMID: 24063569; PubMed Central PMCID: PMC3853622.

15. Capasso L, Gualano MR, Flacco ME, Siliquini R, Manzoli L. E-cigarette regulations in Italy: fluctuating and confusing. Lancet. 2014; 383(9932):1883. Epub 2014/06/03. doi: 10.1016/S0140-6736(14)609089S0140-6736(14)60908-9 [pii]. PMID: 24881982.

16. Sarewitz D. Allow use of electronic cigarettes to assess risk. Nature. 2014; 512(7515):349. Epub 2014/ 08/29. doi: 10.1038/512349a512349a [pii]. PMID: 25164716.

17. Bhatnagar A, Whitsel LP, RibisI KM, Bullen C, Chaloupka F, Piano MR, et al. Electronic Cigarettes: A Policy Statement From the American Heart Association. Circulation. 2014. Epub 2014/08/27. doi: CIR.0000000000000107 [pii]doi: 10.1161/CIR.0000000000000107 PMID: 25156991.

18. Igari $\mathrm{H}$, Segawa $S$, Watanabe $A$, Suzuki $A$, Watanabe $M$, Sakurai $T$, et al. Immunogenicity of a monovalent pandemic influenza A H1N1 vaccine in health-care workers of a university hospital in Japan. Microbiol Immunol. 2010; 54(10):618-24. Epub 2010/12/02. doi: 10.1111/j.1348-0421.2010.00254.x PMID: 21118299.

19. Schraufnagel DE, Blasi F, Drummond MB, Lam DC, Latif E, Rosen MJ, et al. Electronic cigarettes. A position statement of the forum of international respiratory societies. Am J Respir Crit Care Med. 2014; 190(6):611-8. Epub 2014/07/10. doi: 10.1164/rccm.201407-1198PP PMID: 25006874.

20. Drummond MB, Upson D. Electronic cigarettes. Potential harms and benefits. Ann Am Thorac Soc. 2014; 11(2):236-42. Epub 2014/03/01. doi: 10.1513/AnnalsATS.201311-391FR PMID: 24575993.

21. Fairchild AL, Bayer R, Colgrove J. The renormalization of smoking? E-cigarettes and the tobacco "endgame". N Engl J Med. 2014; 370(4):293-5. Epub 2013/12/20. doi: 10.1056/NEJMp1313940 PMID: 24350902.

22. The Lancet O. Time for e-cigarette regulation. Lancet Oncol. 2013; 14(11):1027. Epub 2013/10/02. doi: 10.1016/S1470-2045(13)70468-6S1470-2045(13)70468-6 [pii]. PMID: 24079859.

23. E-cigarettes-aid to smoking cessation or smokescreen? Lancet. 2014; 384(9946):829. Epub 2014/09/ 12. doi: 10.1016/S0140-6736(14)61470-7S0140-6736(14)61470-7 [pii]. PMID: 25209468.

24. Cobb NK, Abrams DB. E-cigarette or drug-delivery device? Regulating novel nicotine products. N Engl J Med. 2011; 365(3):193-5. Epub 2011/07/22. doi: 10.1056/NEJMp1105249 PMID: 21774706.

25. Hajek P, Etter JF, Benowitz N, Eissenberg T, McRobbie H. Electronic cigarettes: review of use, content, safety, effects on smokers and potential for harm and benefit. Addiction. 2014; 109(11):1801-10. Epub 2014/08/01. doi: 10.1111/add.12659 PMID: 25078252.

26. Borland R. Electronic cigarettes as a method of tobacco control. BMJ. 2011; 343:d6269. Epub 2011/10/ 04. doi: 10.1136/bmj.d6269bmj.d6269 [pii]. PMID: 21964546.

27. Etter JF. Should electronic cigarettes be as freely available as tobacco? Yes. BMJ. 2013; 346:f3845. Epub 2013/06/19. doi: 10.1136/bmj.f3845bmj.f3845 [pii]. PMID: 23771039.

28. Burstyn I. Peering through the mist: systematic review of what the chemistry of contaminants in electronic cigarettes tells us about health risks. BMC Public Health. 2014; 14:18. Epub 2014/01/11. doi: 10. 1186/1471-2458-14-181471-2458-14-18 [pii]. PMID: 24406205; PubMed Central PMCID: PMC3937158.

29. Abrams DB. Promise and peril of e-cigarettes: can disruptive technology make cigarettes obsolete? JAMA. 2014; 311(2):135-6. Epub 2014/01/09. doi: 10.1001/jama.2013.2853471812971 [pii]. PMID: 24399548.

30. Gualano MR, Passi S, Bert F, La Torre G, Scaioli G, Siliquini R. Electronic cigarettes: assessing the efficacy and the adverse effects through a systematic review of published studies. J Public Health (Oxf). 2014. Epub 2014/08/12. doi: fdu055 [pii]doi: 10.1093/pubmed/fdu055 PMID: 25108741. 
31. Krall EA, Garvey AJ, Garcia RI. Smoking relapse after 2 years of abstinence: findings from the VA Normative Aging Study. Nicotine Tob Res. 2002; 4(1):95-100. Epub 2002/03/22. doi: 10.1080/ 14622200110098428 PMID: 11906685.

32. Chen IL. FDA summary of adverse events on electronic cigarettes. Nicotine Tob Res. 2013; 15(2):6156. Epub 2012/08/03. doi: 10.1093/ntr/nts145nts145 [pii]. PMID: 22855883. 